\title{
Repulsive depletion interactions in colloid-polymer mixtures
}

\author{
Daniel Rudhardt, Clemens Bechinger and Paul Leiderer \\ Fakultät für Physik, University of Konstanz, D-78457 Konstanz, Germany
}

Received 21 July 1999

\begin{abstract}
Depletion forces in colloidal systems are known to be entirely attractive, as long as the background of macromolecules is small enough that an ideal gas approach is valid. At higher densities, however, structural correlation effects of the macromolecules which lead to additional repulsive parts in the depletion interaction, have to be taken into account. We have measured the depletion interaction between a single polystyrene sphere and a wall in the presence of non-ionic polymer coils. Although the polymer volume fraction is very small, our results clearly show the existence of repulsive parts, leading to a large over-estimation of correlation effects in a hard sphere liquid. The possible explanation of this discrepancy by additionally considering the role of van der Waals forces is discussed.
\end{abstract}

\section{Introduction}

The ability of non-adsorbing polymers to promote flocculation in colloidal suspensions has been well known since 1925 when Traube observed the phase separation of natural rubbers by the addition of water-soluble polymers [1]. Since the stability of mixed systems is an important aspect in a variety of industrial and agricultural processes, in the meantime numerous other researchers have studied this phenomenon, often referred to as depletion interaction [2]. The first satisfactory explanation of depletion forces was given by Asakura and Oosawa (AO) [3] in 1954. Following their arguments, depletion forces can be understood by considering two large colloidal spheres of radius $R$ in the presence of a hard sphere fluid of smaller particles with radius $r$ (referred to as macromolecules in the following). When the distance between the two spheres is smaller than the diameter of the macromolecules the region between the particles becomes depleted with respect to the macromolecule concentration $n$. Such a concentration gradient, however, leads to an osmotic pressure causing an effective attraction between the colloidal spheres. The same effect occurs also when one sphere is replaced by a flat wall.

In the simple AO theory the macromolecules are considered as non-interacting particles (ideal gas approximation) which is only justified when the density $n$ of the macromolecules is small. For increasing $n$, however, structural correlation effects which lead to an oscillatory density profile of the macromolecule liquid close to the wall, have to be taken into account. As has been pointed out recently, this gives rise to a strong modification of the depletion potential experienced by a large colloidal sphere $[4,5]$. As a result, depletion forces are no longer entirely attractive as predicted by the $\mathrm{AO}$ theory, but show also repulsive contributions. The latter effect is strongly increased when the size ratio between the colloids and the macromolecules becomes large.

Indeed, such long-ranged oscillating depletion forces are experimentally confirmed in systems of charged macromolecules where the contributions of correlation effects among the 
macromolecules are pronounced [6-9]. Very recently, repulsive depletion forces have also been observed in binary hard sphere mixtures [10].

In this work we have measured depletion potentials of a single colloidal sphere in front of a glass surface in the presence of non-ionic macromolecules for various macromolecule densities. In contrast to previous experiments [11], here we have increased the $R / r$ ratio by almost a factor of five and find clear evidence for the occurrence of both attractive and repulsive interaction energies.

\section{Experiment}

We used total internal reflection microscopy (TIRM) $[12,13]$ to measure the potential of a colloidal sphere immersed in a fluid of coiled, non-ionic polymer chains close to a glass surface.

The method of TIRM is based on the effect of frustrated total internal reflection. When a laser beam is reflected at an angle $\theta>\theta_{C}$ (with $\theta_{C}$ being the critical angle of total internal reflection) at the interface between a glass plate and the colloidal suspension, an evanescent light field is created which leaks into the suspension. The intensity of the evanescent field is well known to decay exponentially with a penetration depth $\beta^{-1}$, which is a function of $\theta$ and the refractive indices of the materials comprising the interface. Under our experimental conditions $\beta^{-1}$ is typically of the order of magnitude of the wavelength of the light beam. When a colloidal sphere approaches the surface close enough to enter the evanescent field frustrated total reflection will occur and the sphere will scatter the evanescent field. The scattering intensity $I$ of the colloidal sphere has been shown to be proportional to that of the evanescent wave and thus can be written as $I=I_{0} \mathrm{e}^{-\beta z}$ [14]. Measuring $I$, which fluctuates due to Brownian motion, of the sphere perpendicular to the surface as a function of time thus provides a sensitive and non-intrusive method to determine the distance $z$ between the sphere and the wall. In thermal equilibrium the probability distribution $p(z)$ of finding the particle a certain distance to the wall is given by the Boltzmann distribution, which allows us to calculate the overall interaction potential $\Phi(z)$ from the measured scattering signal. For details regarding TIRM we refer to the literature and our previous work $[11-13,15,16]$.

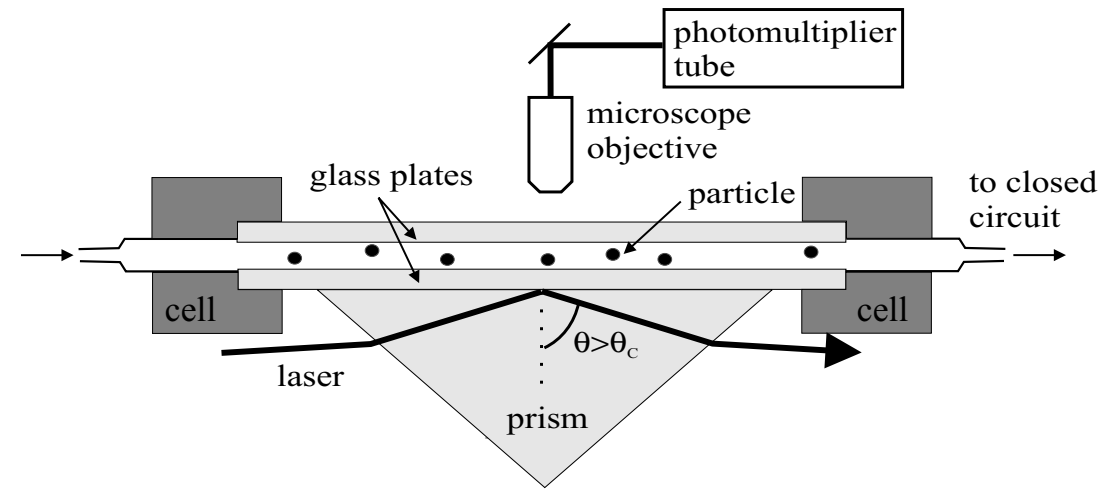

Figure 1. Schematic TIRM setup used in our experiment.

Figure 1 shows a schematic representation of our TIRM setup. A cell was composed of two fused silica glass plates separated by a spacer $(d=1 \mathrm{~mm}$, not shown). After assembling the cell, a BK7 prism was optically matched to one of the glass plates. Then 
the cell was connected to a closed circuit which contained the colloidal suspension. We used monodisperse polystyrene (PS) spheres with radius $R=5 \mu \mathrm{m}$ which were suspended in water. The particles were charge stabilized with sulphate surface groups. Only highly diluted suspensions with a particle density less than $0.5 \mathrm{~mm}^{-3}$ were used to guarantee that only a single particle was in the field of view and contributed to the scattering signal. The suspension was pumped through a closed circuit which contained an ion exchange resin and an electrical conductivity probe to control the ionic strength of the suspension [17]. After adjusting the desired ionic strength a given amount of polyethylene oxide (PEO) with a molecular weight $M_{W}=1 \times 10^{6}$ was added. Only in diluted solutions could the polymer coils be considered as isolated spherical particles. The transition between a diluted and a semi-diluted solution can be described by the critical density $n^{*}$ [18]. The maximum polymer concentration used in our experiments was $23.4 \mu \mathrm{m}^{-3}$, which is a factor of 30 below the critical concentration $n^{*}$. The radius of gyration of these polymer coils can be determined by static light scattering and was found to be $R_{G}=67.7 \mathrm{~nm}$ for the molecular weight of PEO used in our experiments [19].

The scattered intensity was collected by a microscope objective lens (Plan L50×, Leitz) and focused onto a photomultiplier tube. To obtain sufficient data and to minimize statistical errors the scattering intensity of a particle was sampled over at least $1000 \mathrm{~s}$ at a sampling rate of $50 \mathrm{~Hz}$.

\section{Results and discussion}

Depletion forces which act on a single colloidal particle suspended in a liquid of macromolecules are typically in the range of nano-to piconewtons. Therefore, one has to assure that the method of TIRM is sensitive enough to detect such small forces in our experiments. To determine the force resolution in our setup in the following we first measured the particle-wall potential of single colloids (in the absence of macromolecules) with slightly different weights but with the same size and surface charge.

The potential of a negatively charged PS particle above a glass plate is well known to be composed of two parts: towards smaller distance the potential increases exponentially owing to the electrostatic repulsion between the sphere and the fused silica plate which is also negatively charged when in contact with water. For larger distances the potential is completely dominated by the weight of the particle. Accordingly the particle-wall potential can be written as [12]

$$
\frac{\Phi(z)-\Phi_{0}}{k_{B} T}=\frac{A}{k_{B} T} \mathrm{e}^{-\kappa z}+\frac{G}{k_{B} T} z
$$

where $A$ is a constant which depends on the surface potential of the sphere and the wall, $\kappa$ is the Debye screening length and $G=\left(\rho_{P}-\rho_{S}\right) V g$ is the weight of the particle with $\rho_{P}$ and $\rho_{S}$ being the density of PS and the solvent, respectively, $V$ the particle volume and $g$ the acceleration of gravity.

Variation of $G$ was achieved by adding different amounts of deuterated water $\left(\mathrm{D}_{2} \mathrm{O}\right)$ to the suspension, which increases the buoyancy the particle and thus reduces its weight. By increasing the heavy water concentration successively we obtained different potential curves which are plotted as symbols in figure 2. From the $\mathrm{D}_{2} \mathrm{O} / \mathrm{H}_{2} \mathrm{O}$ concentration the effective weight of the particles was calculated to be $255 \mathrm{fN}$ (a), $197 \mathrm{fN}($ b), $138 \mathrm{fN}(\mathrm{c}), 85 \mathrm{fN}$ (d) and $40 \mathrm{fN}$ (e), respectively. The solid lines correspond to calculations according to equation (1), where the above values for $G$ were used. The Debye length was obtained from the measured electrical conductivity of the suspension and the parameter $A$ was determined from the minimum position of the potential curve corresponding to the experiment without heavy water (a). The potential 


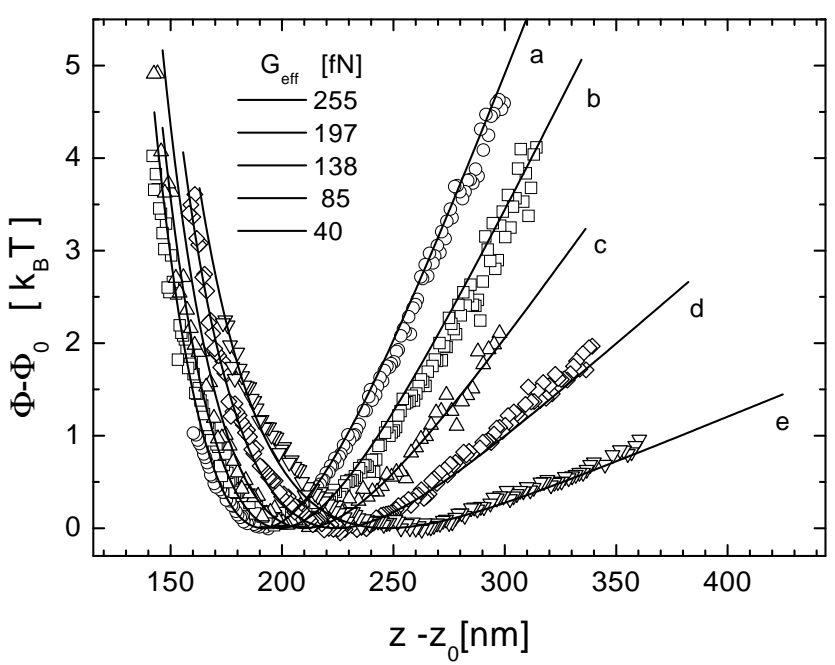

Figure 2. Measured interaction potentials (symbols) of a PS sphere $(R=5 \mu \mathrm{m})$ and a flat surface for different heavy water concentrations. The solid lines are calculations according to equation (1).

curves b-e are only known as a function of relative distances, since the scattering intensity at particle wall contact was not determined for these measurements. Therefore we plotted these curves in such a way that their minimum positions coincide with that of the theoretical predictions.

As expected, the slope of the gravitational part of the experimental potential curves decreases with increasing concentration of heavy water, which is also in good agreement with the theoretical predictions. As can be seen by comparing curves $\mathrm{d}$ and e, which correspond to a difference in the weight force of $45 \mathrm{fN}$, the resolution of our method is clearly below this value. In fact, we estimated the force resolution of our TIRM setup to be of the order of $10 \mathrm{fN}$. This demonstrates that the method of TIRM is able to measure entropic forces in colloidal systems.

When PEO is added to the system the potentials are altered due to the occurrence of depletion forces. This is shown in figure 3 which corresponds to polymer concentrations $n=0$ (a), $4.1 \mu \mathrm{m}^{-3}$ (b), $8.7 \mu \mathrm{m}^{-3}$ (c), $16.4 \mu \mathrm{m}^{-3}$ (d) and $23.4 \mu \mathrm{m}^{-3}$ (e), respectively. For clarity the potentials are separated in the vertical direction by $3 k_{B} T$. With increasing polymer concentration we observe that the potential minimum is shifted towards smaller distances and becomes deeper. A similar effect has been also observed in our previous experiments [11] with smaller $R / r$ ratio and is characteristic for depletion forces. Additionally, however, we find a local maximum around $z=150 \mathrm{~nm}$ which increases as the PEO concentration is increased. This becomes more obvious when the gravitational and electrostatic part of the potential (according to equation (1)) is subtracted. This was done exemplarily for curve 3c and replotted as an inset in figure 3, which clearly demonstrates the existence of a potential barrier of about $0.75 k_{B} T$.

It has been mentioned already that such potential barriers are expected when the density of macromolecules is high and their concentration close to the wall exceeds the bulk density. According to Mao et al [4], the potential barrier $W$ can be approximated by

$$
W=1.2 \frac{R}{r} \Phi^{2}
$$

If we calculate this expression for a volume fraction of $\Phi=4.2 \%$ which corresponds to 


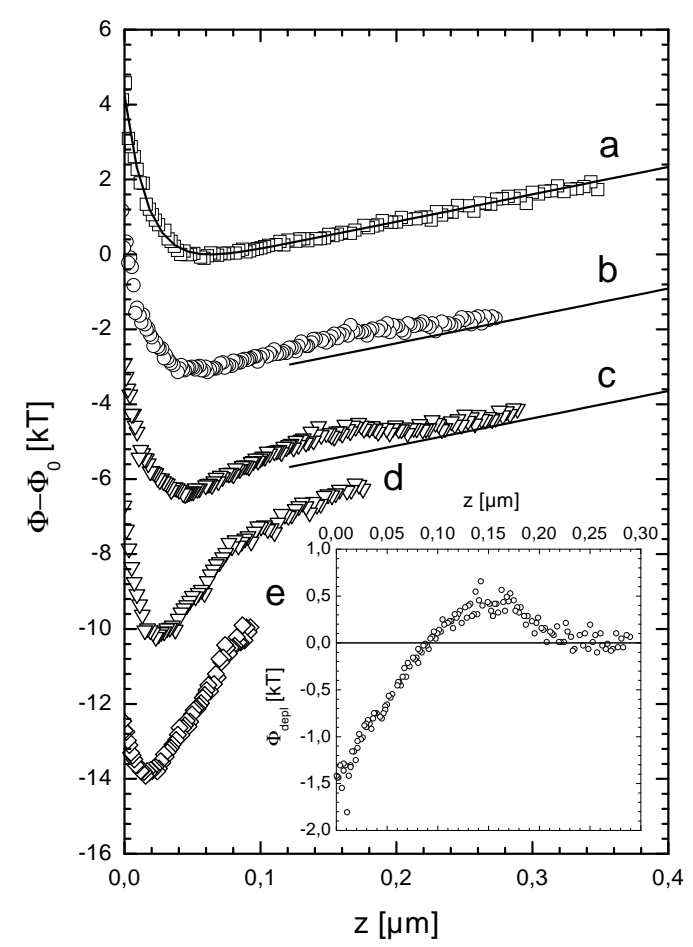

Figure 3. Measured interaction potentials (symbols) of a PS sphere ( $R=5 \mu \mathrm{m}$ ) and a flat surface for polymer concentrations $n=0$ (a), $4.1 \mu \mathrm{m}^{-3}$ (b), $8.7 \mu \mathrm{m}^{-3}$ (c), $16.4 \mu \mathrm{m}^{-3}$ (d) and $23.4 \mu \mathrm{m}^{-3}$ (e), respectively. The solid line (a) is the calculation according to equation (1). The straight lines, which have the same slope as the linear part of curve a are plotted as a guide to the eye. The inset is a replot of curve $\mathrm{c}$ after subtracting the electrostatic and gravitational part of the potential.

curve $3 \mathrm{c}$, we obtain a potential barrier of only $0.1 \mathrm{kT}$, which is too small to account for our experimental observations. Additionally, the ratio between the potential barrier and the contact value of the potential at $z=0$ is too small to be explained by these theoretical predictions which are based on the assumptions of hard sphere systems.

Even in systems of uncharged macromolecules, however, a hard sphere and hard wall model does not always strictly apply due to the existence of van der Waals forces which increase as $1 / z^{3}$ [20]. Accordingly, this may lead to a change of the density profile of the macromolecules at small distances below $150 \mathrm{~nm}$. Indeed, such a mechanism would increase the PEO density close to the surface and thus lead even at small polymer concentrations to a smaller depletion potential at $z=0$ and a more pronounced repulsive barrier as observed in our experiments.

Finally we want to address the question of why the influence of van der Waals forces was not observed in our previous experiments where PS spheres of $3 \mu \mathrm{m}$ radius and PEO with a smaller radius of gyration, i.e. $r_{G}=101 \mathrm{~nm}$ was used. Due to the greatly increased weight of the PS particles and the smaller Debye screening length in the present study, our present measurements are much more sensitive to changes in the particle-wall potential below $z=150 \mathrm{~nm}$. In the earlier experiments, this range was dominated by the electrostatic repulsion effects between the PS sphere and the wall. Calculations on the basis of density functional theory are in progress which include van der Waals forces to elucidate their influence on repulsive depletion interactions. 


\section{Conclusions}

Our measurements show that repulsive depletion interaction may occur between a colloidal sphere and a flat wall even in the presence of very diluted uncharged macromolecules. Repulsive contributions to the depletion interaction are predicted by recent theories but are too small to explain our experimental data. We suggest that the consideration of van der Waals forces between the macromolecules and the surface may explain this discrepancy between theory and experiment.

\section{Acknowledgments}

We would like to acknowledge helpful discussions with Roland Roth. This work was supported by the SFB 513 from the Deutsche Forschungsgemeinschaft and the Optikzentrum Konstanz.

\section{References}

[1] Traube J 1925 Gummi-Ztg. 39 434-5

[2] Russel W B, Saville D A and Schowalter W R 1989 Colloidal Dispersions (Cambridge: Cambridge University Press)

[3] Asakura S and Oosawa F 1954 J. Chem. Phys. 22 1255-6

[4] Mao Y, Cates M E and Lekkerkerker H N W 1995 Physica A 222 10-24

[5] Götzelmann B, Evans R and Dietrich S 1998 Phys. Rev. E 57 6785-800

[6] Sharma A and Walz J Y 1996 J. Chem. Soc., Faraday Trans. 92 4997-5004

[7] Sober D L and Walz J Y 1995 Langmuir 11 2352-6

[8] Richetti P and Kekicheff P 1992 Phys. Rev. Lett. 68 1951-4

[9] Milling A J and Vincent B 1997 J. Chem. Soc., Faraday Trans. 93 3179-83

[10] Crocker J C, Matteo J A, Dinsmore A D and Yodh A G 1999 Phys. Rev. Lett. $824352-5$

[11] Rudhardt D, Bechinger C and Leiderer P 1998 Phys. Rev. Lett. 81 1330-3

[12] Prieve D C, Luo F and Lanni F 1987 Faraday Discuss. Chem. Soc. 83 297-307

[13] Walz J Y 1997 Current Opinion Colloidal Interfaces Sci. 2600

[14] Prieve D C and Walz J Y 1993 Appl. Opt. 32 1629-41

[15] Rudhardt D, Bechinger C and Leiderer P 1998 Prog. Colloid Polym. Sci. $11037-40$

[16] Rudhardt D, Bechinger C and Leiderer P 1999 Prog. Colloid Polym. Sci. 112 163-7

[17] Palberg T, Härtl W, Wittig U, Versmold H, Würth M and Simnacher E 1992 J. Phys. Chem. 96 8180-3

[18] de Gennes P-G 1988 Scaling Concepts in Polymer Physics (New York: Cornell University Press)

[19] Devanand K and Selser J C 1991 Macromolecules 24 5943-7

[20] Israelachvili J N 1985 Intermolecular and Surface Forces (London: Academic) 